\title{
Asynchronous Exponential Growth of a General Structured Population Model
}

\author{
Jacek Banasiak • Katarzyna Pichór · Ryszard Rudnicki
}

Received: 1 April 2011 / Accepted: 17 December 2011 / Published online: 30 December 2011

(C) The Author(s) 2011. This article is published with open access at Springerlink.com

\begin{abstract}
We consider a class of structured cell population models described by a first order partial differential equation perturbed by a general birth operator which describes in a unified way a wide class of birth phenomena ranging from cell division to the McKendrick model. Using the theory of positive stochastic semigroups we establish new criteria for an asynchronous exponential growth of solutions to such equations.
\end{abstract}

Keywords Structured population · Fragmentation equation · First order partial differential equation · Positive semigroups · Asymptotic stability

Mathematics Subject Classification (2010) Primary 47D06 · Secondary 45K05 · 92D25

\section{Introduction}

Many physical and biological processes can be described in the following way. We have collection of objects called individuals which are characterized by some parameter $m$ which

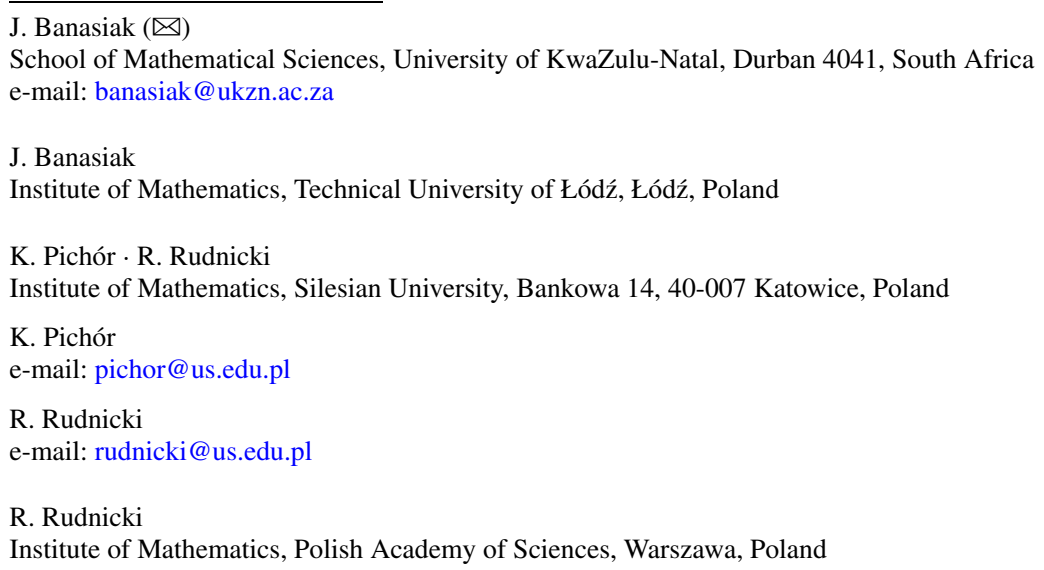


ranges from $m=a$ to $m=1$. The parameter $m$ grows in time according to the equation

$$
\frac{d m}{d t}=g(m)
$$

Moreover, an individual can divide into, or give a birth to, new individuals. We denote by $b_{k}(m), k=1,2, \ldots$, the rates at which an individual with a parameter $m$ produces $k$ individuals. If such an individual produces $k$ individuals, then by $\mathcal{P}_{k}(m, A)$ we denote the probability that its descendant has the parameter located in the set $A$. Indeed, $\mathcal{P}_{k}$ is a transition probability function, i.e. for each $m \in[a, 1]$ the function $A \mapsto \mathcal{P}_{k}(m, A)$ defined on the $\sigma$-algebra $\Sigma$ of Borel subsets of $[a, 1]$ is a probabilistic measure and for each $A \in \Sigma$ the function $m \mapsto \mathcal{P}_{k}(m, A)$ is $\Sigma$-measurable. Then the individual with the parameter $m$ has

$$
\mathcal{P}(m, A)=\sum_{k=1}^{\infty} k b_{k}(m) \mathcal{P}_{k}(m, A)
$$

descendants with parameters in the set $A$ in a unit of time and

$$
b(m)=\sum_{k=0}^{\infty} k b_{k}(m)
$$

is the mean number of its descendants in a unit of time. It is obvious that $\mathcal{P}(m, A) \leq b(m)$ for each $m$ and each $A \in \Sigma$. Further, by $\mu(m)$ we denote the rate of loss of individuals with parameter $m$ and we include in this rate the loss by death or by division. We mention that the technical assumptions, summarized in Sect. 2, ensure that the rates $b$ and $\mathcal{P}$ are finite apart from possibly at $m=1$ and thus the operators introduced below are well defined.

This general approach allows us to investigate different processes usually treated separately. For example, if we consider the process of cell division into two daughter cells, then $\mathcal{P}(m, A)=2 b_{2}(m) \mathcal{P}_{2}(m, A)$ but the transition function $\mathcal{P}_{2}(m, A)$ depends on the choice of the parameter $m$ even in the case of equal division. If $m$ is the volume of a cell, then

$$
\mathcal{P}_{2}\left(m,\left\{\frac{m}{2}\right\}\right)=1,
$$

but if $m$ is the age of a cell, then

$$
\mathcal{P}_{2}(m,\{0\})=1 \text {. }
$$

In the case of unequal division the transition function can be given by a stochastic kernel, i.e.,

$$
\mathcal{P}_{2}(m, A)=\int_{A} k(y, m) d y,
$$

where $k$ is a nonnegative measurable function such that $\int_{a}^{1} k(y, m) d y=1$ for all $m$.

One of the areas in which such models have been used is that of cell replication and maturation and these applications have been around for over 50 years, [6, 21, 22, 27, 31, 32]. A survey of many applications is given in the book edited by Metz and Diekmann [20]. Our model includes models of a population of organisms reproducing by binary fission with equal $[8,10,12,16,17]$ and unequal division $[2,11,13,15]$. Both types of binary fission models were considered in [29]. Moreover, our general scheme includes the classical age structured Sharpe-Lotka-McKendrick model [18, 30].

Our aim is to study the function $N(t, m)$ which describes the distribution of the population with respect to $m$; in other words, the number of individuals with the parameter $m$ 
between $m_{1}$ and $m_{2}$ at a time $t$ is given by the formula $\int_{m_{1}}^{m_{2}} N(t, m) d m$. The equation satisfied by the function $N$ depends on the choice of the transition function $\mathcal{P}$. For instance, if $\mathcal{P}$ is of the form (5), then $N(t, m)$ satisfies a first order partial differential equation with an integral boundary condition while in the cases (4) and (6) we obtain also a first order partial differential equation but with an integral perturbation.

In order to unify the description of our process it is better to begin with the dual (backward) equation

$$
\frac{\partial u}{\partial t}=g(m) \frac{\partial u}{\partial m}-\mu(m) u(t, m)+\int_{a}^{1} u(t, y) \mathcal{P}(m, d y) .
$$

Let $b^{a}(m)=\mathcal{P}(m,\{a\})$, i.e. $b^{a}(m)$ describes the rate at which the individuals with the parameter $m$ produce individuals with the minimal parameter $a$, like in the age-structured population model. We denote by $\mathcal{P}^{r}$ the remaining part of the transition function $\mathcal{P}$, i.e. $\mathcal{P}^{r}(m, A)=\mathcal{P}(m, A \backslash\{a\})$ and we assume that for each $m$ the measure $\mathcal{P}^{r}(m, \cdot)$ is absolutely continuous with respect to the Lebesgue measure for a.e. $m$. Further, let $b^{r}(m)=$ $\mathcal{P}^{r}(m,[a, 1])$. Then, by the Radon-Nikodym theorem, there exists an operator $P$ defined on the space

$$
L_{b}^{1}[a, 1]=\left\{f \in L^{1}[a, 1]: \int_{a}^{1}|f(m)| b^{r}(m) d m<\infty\right\}
$$

and with values in the space $L^{1}[a, 1]$ such that for each nonnegative function $f \in L_{b}^{1}[a, 1]$ and each set $A \in \Sigma$ we have

$$
\int_{A} P f(y) d y=\int_{a}^{1} \mathcal{P}^{r}(m, A) f(m) d m .
$$

If the distribution function $N(t, m)$ is sufficiently regular, then it satisfies the following equation

$$
\frac{\partial N}{\partial t}+\frac{\partial(g(m) N)}{\partial m}=-\mu(m) N(t, m)+P N(t, m)
$$

with the boundary condition

$$
g(a) N(t, a)=\int_{a}^{1} b^{a}(m) N(t, m) d m
$$

and with the initial condition

$$
N(0, m)=N_{0}(m) \text { for } m \in[a, 1] .
$$

The main aim of this paper is to reduce the problem to a Markov one by using the Perron eigenvectors of (7) and (9) and then to apply the techniques of stochastic semigroups [19, $25,26,28,29]$ to study asynchronous exponential growth (AEG) property of the system (9)-(11). The idea of using Perron eigenvectors is not new and has been successfully used for instance in $[23,24]$, where it was combined with the relative entropy technique to prove the exponential decay of solutions. The stochastic semigroups techniques, used in this paper, allow us to consider quite general singular growth and death rates as opposed to [24] but, on the other hand, places some restrictions on the birth process. Thus our results are complementary to those of $[23,24]$. We note that instead one also can use known results on compact semigroups but this would require analyzing the whole spectrum of the generator which is rather difficult. In our method no compactness properties of the solution semigroup are required. 
The most difficult part of the reduction of the problem to a Markov problem, which is the cornerstone of our method, is to check that the Perron eigenvector of the adjoint equation is bounded and separated from zero. It is then interesting to note that even a nonlinear model of cell population (cf. [17]) can be reduced to a Markov semigroup.

\section{Formulation of the Results}

We collect all assumptions concerning our model. We assume that $g:[a, 1] \rightarrow(0, \infty)$ is a continuously differentiable function (hence, in particular, $g$ is bounded away from zero), $\mu:[a, 1) \rightarrow[0, \infty)$ and $b_{k}:[a, 1) \rightarrow[0, \infty)$ are continuous functions. Further we assume that for every $\bar{m} \in(a, 1)$

$$
\int_{\bar{m}}^{1} \mathcal{P}(m,[a, \bar{m})) d m>0 .
$$

This assumption has the following biological interpretation: for every $\bar{m} \in(a, 1)$ there is a mother with maturity greater than $\bar{m}$ which has daughters with maturity less than $\bar{m}$. It means that if (12) was not satisfied, then for some $\bar{m}$ all daughters of any mother with $m \geq \bar{m}$ also would have maturity greater than $\bar{m}$ and thus we could restrict the set of parameters to the interval $[\bar{m}, 1]$.

We also assume that a mother of maturity $m$ cannot have daughters with maturity exceeding $m-h$, that is,

$$
\mathcal{P}(m,[a, m-h])=1 \quad \text { for all } m \in[a, 1] .
$$

Condition (13) can be rewritten in an equivalent form

$$
\mathcal{P}(m,(m-h, 1])=0 \quad \text { for all } m \in[a, 1] .
$$

From (14) and the definition of $b$ it follows in particular that $b(m)=0$ for $m \in[a, a+h]$. We assume that $\inf \{b(m): m \in[1-h, 1)\}>0$, which means that any cell with maturity $\geq 1-h$ can divide. Another immediate consequence of $(13)$ is that $(P f)(m)$ depends only on values of $f$ in $[m+h, 1]$ and, in particular, $P f=0$ on $[1-h, 1]$. Since the maximal value of the parameter $m$ is 1 , we have to assume that $\int_{a}^{1} \mu(m) d m=\infty$, that is, all cells die or divide before or at reaching maturity 1 . Moreover, there is a constant $\bar{C}>0$ such that

$$
\bar{C}^{-1} \mu(m) \leq b(m) \leq \bar{C} \mu(m) \text { for } m \geq 1-h .
$$

Remark 1 The assumption (15) is natural because $\mu(m)$ includes the rate of loss by division. For example if we consider only the division into two cells then $\mu(m)=\mu_{0}(m)+b_{2}(m)$ and $b(m)=2 b_{2}(m)$, where $\mu_{0}(m)$ is the mortality rate and $b_{2}(m)$ is the division rate. In this case we can assume that $\mu_{0}(m)$ is a bounded function and $b_{2}(m)$ is a strictly positive function for $m \geq 1-h$, which implies (15).

Let $P$ be defined by (8) and denote by $A_{b^{a}}$ the differential operator $-(g N)^{\prime}-\mu N$ restricted to the domain

$$
D\left(A_{b^{a}}\right)=\left\{N \in L^{1}[a, 1] \cap A C(a, 1): \mu N,(g N)^{\prime} \in L^{1}[a, 1], N \text { satisfies (10) }\right\},
$$

where $A C(a, 1)$ denotes the space functions which are absolutely continuous on each compact subinterval of $[a, 1)$. 
Theorem 1 The operator $\left(A_{b^{a}}+P, D\left(A_{b^{a}}\right)\right)$ generates a strongly continuous positive semigroup, denoted $\{T(t)\}_{t \geq 0}$, on $L^{1}[a, 1]$.

The proof of Theorem 1 is given in Sect. 3. The main result of the paper is the following theorem on asynchronous exponential growth of the population.

Theorem 2 Assume that one of the following conditions holds:

(I) there exists a measurable function $q:[a, 1] \times[a, 1] \rightarrow[0, \infty)$ such that

$$
\int_{a}^{1} \int_{a}^{1} q(y, m) d m d y>0 \quad \text { and } \quad \mathcal{P}(m, A) \geq \int_{A} q(y, m) d y
$$

for any $m \in[a, 1]$ and $A \in \Sigma$,

(II) there exist $m_{0} \in(a, 1), \varepsilon>0$ such that $b^{a}(m)>0$ for $m \in\left(m_{0}-\varepsilon, m_{0}+\varepsilon\right)$,

(III) there exist $m_{0} \in(a, 1), \varepsilon>0$ and a $C^{1}$-function $r:\left(m_{0}-\varepsilon, m_{0}+\varepsilon\right) \rightarrow[a, 1]$ such that $g\left(r\left(m_{0}\right)\right) \neq r^{\prime}\left(m_{0}\right) g\left(m_{0}\right)$ and

$$
\mathcal{P}(m,\{r(m)\}) \geq \varepsilon \quad \text { for } m \in\left(m_{0}-\varepsilon, m_{0}+\varepsilon\right) .
$$

Then there exist a continuous function $f_{*}:[a, 1] \rightarrow[0, \infty), f_{*}(m)>0$ for $m \in(a, 1)$ and a continuous function $w:[a, 1] \rightarrow(0, \infty)$ such that $e^{-\lambda t} N(t, \cdot)$ converges in $L^{1}[a, 1]$ to $f_{*} \int_{a}^{1} N(0, m) w(m) d m$.

Condition (I) amounts to $m \mapsto \mathcal{P}^{r}(m, \cdot)$ being not zero almost everywhere. The proof of Theorem 2 is given in Sects. 5 and 6. It should be noted that in some special cases none of conditions I, II, III holds. For example, if $m$ is the weight of a cell, $g(m)=m$ and we have equal division, that is, $\mathcal{P}(m, A)=2$ if $m / 2 \in A$ and $\mathcal{P}(m, A)=0$ if $m / 2 \notin A$. Then the weight of daughter cells at any given time does not depend on the moment of division. In this case the distribution of masses of daughters strongly depends on the initial distribution. Of course in this case the semigroup $\{T(t)\}_{t \geq 0}$ has no asynchronous exponential growth. An interesting example of a linear population model which has no asynchronous exponential growth property is a model with telomere loss [3].

\section{Proof of Theorem 1}

Consider the 'formal' equation for the resolvent of $A_{b^{a}}$,

$$
\lambda N(m)+(g(m) N(m))^{\prime}+\mu(m) N(m)=f(m),
$$

whose solution is given by

$$
N_{\lambda}(m)=\frac{e^{-\lambda G(m)-Q(m)}}{g(m)} \int_{a}^{m} e^{\lambda G(s)+Q(s)} f(s) d s+C \frac{e^{-\lambda G(m)-Q(m)}}{g(m)},
$$

where $G(m)=\int_{a}^{m}(1 / g(s)) d s$ and $Q(m)=\int_{a}^{m}(\mu(s) / g(s)) d s$. To shorten notation we denote

$$
e_{-\lambda}(m):=e^{-\lambda G(m)-Q(m)}, \quad e_{\lambda}(m):=e^{\lambda G(m)+Q(m)}
$$

and note two useful formulae. For $s \in[a, 1)$

$$
\int_{s}^{1} \frac{e^{-\lambda G(m)}}{g(m)} d m=-\frac{1}{\lambda} \int_{s}^{1} \frac{d}{d m} e^{-\lambda G(m)} d m \leq \frac{e^{-\lambda G(s)}}{\lambda}
$$


and, adding and subtracting $\lambda$ in the numerator below and noting that $e_{-\lambda}(1)=0$, similarly we obtain

$$
\int_{s}^{1} \frac{\mu(m) e_{-\lambda}(m)}{g(m)} d m=e_{-\lambda}(s)-\lambda \int_{s}^{1} \frac{e_{-\lambda}(m)}{g(m)} d m .
$$

Returning to (18) and arguing as in [5] with help of (19) and (20) we see that the first term in (18)

$$
N_{\lambda, 0}(m)=\frac{e_{-\lambda}(m)}{g(m)} \int_{a}^{m} e_{\lambda}(s) f(s) d s
$$

is the resolvent $R\left(\lambda, A_{0}\right)$ of the operator $A_{0}$ corresponding to the homogeneous Dirichlet boundary condition at $m=a$ and it satisfies

$$
\begin{aligned}
\int_{a}^{1} N_{\lambda, 0}(m) d m & \leq \frac{1}{\lambda}\|f\|, \quad \lambda>0, \\
\int_{a}^{1} \mu(m) N_{\lambda, 0}(m) d m & \leq\|f\| .
\end{aligned}
$$

We introduce the notation $\langle f, g\rangle:=\int_{a}^{1} f(m) g(m) d m$. To deal with the boundary term, we let $m \rightarrow a$ in (18) and, using the boundary condition, we obtain

$$
N_{\lambda}(m)=N_{\lambda, 0}(m)+\frac{e_{-\lambda}(m)\left\langle b^{a}, N_{\lambda}\right\rangle}{g(m)}
$$

which can be solved for $\left\langle b^{a}, N_{\lambda}\right\rangle$, yielding

$$
\left\langle b^{a}, N_{\lambda}\right\rangle=\frac{\left\langle b^{a}, N_{\lambda, 0}\right\rangle}{1-\left\langle b^{a}, g^{-1} e_{-\lambda}\right\rangle},
$$

provided $\Psi(\lambda):=\left\langle b^{a}, g^{-1} e_{-\lambda}\right\rangle<1$. Let us consider this condition. Using (15), we see that $\Psi(\lambda) \leq \bar{C} \int_{a}^{1} \mu(s) \frac{e_{-\lambda}(s)}{g(s)} d s$. Since $\mu e_{-\lambda} / g \leq \mu e^{-Q} / g$, with the latter function integrable by (20), the Lebesgue dominated convergence theorem yields $\Psi(\lambda) \rightarrow 0$ as $\lambda \rightarrow \infty$. Thus, for some $\bar{\lambda}$ and $b_{\infty}<1,\left\langle b^{a}, g^{-1} e_{-\lambda}\right\rangle \leq b_{\infty}$ for all $\lambda>\bar{\lambda}$ and for such $\lambda$, using (19) with $s=a$ and (22), we have the estimate

$$
\left\|N_{\lambda}\right\| \leq\left\|N_{\lambda, 0}\right\|+\frac{1}{\lambda}\left|\frac{\left\langle b^{a}, N_{\lambda, 0}\right\rangle}{1-\left\langle b^{a}, g^{-1} e_{-\lambda}\right\rangle}\right| \leq\left\|N_{\lambda, 0}\right\|+\frac{\bar{C}}{\lambda} \frac{\|f\|}{1-b_{\infty}} \leq \frac{1+\bar{C}-b_{\infty}}{\left(1-b_{\infty}\right) \lambda}\|f\| .
$$

By the previous calculations $\mu e_{-\lambda} / g$ is integrable and thus $R\left(\lambda, A_{b^{a}}\right)$ for $\lambda>\bar{\lambda}$ is defined by

$$
R\left(\lambda, A_{b^{a}}\right) f=R\left(\lambda, T_{0}\right) f+\frac{e_{-\lambda}}{g} \frac{\left\langle b^{a}, R\left(\lambda, T_{0}\right) f\right\rangle}{1-\left\langle b^{a}, g^{-1} e_{-\lambda}\right\rangle} .
$$

Next we prove that for any $\lambda>0$

$$
\lim _{m \rightarrow 1^{-}} e_{\lambda}(m) \int_{m}^{1} \frac{e_{-\lambda}\left(m^{\prime}\right)}{g\left(m^{\prime}\right)} d m^{\prime}=0
$$

Indeed, consider the family of functionals $\left\{\xi_{m}\right\}_{m \in(1-\epsilon, 1]}$ for some $\epsilon>0$ defined by

$$
\xi_{m} f=e_{\lambda}(m) \int_{m}^{1} \frac{e_{-\lambda}\left(m^{\prime}\right)}{g\left(m^{\prime}\right)} f\left(m^{\prime}\right) d m^{\prime}
$$

for $f \in L^{1}[a, 1]$. We have

$$
\left|\xi_{m} f\right| \leq e_{\lambda}(m) \int_{m}^{1} \frac{e_{-\lambda}\left(m^{\prime}\right)}{g\left(m^{\prime}\right)}\left|f\left(m^{\prime}\right)\right| d m^{\prime} \leq c_{g} \int_{a}^{1}\left|f\left(m^{\prime}\right)\right| d m^{\prime}
$$


on account of monotonicity of $e_{\lambda}$ with $c_{g}=1 / \inf _{m \in[a, 1]} g(m)<+\infty$ by assumption. Moreover, for $f$ with support in $[a, 1-\delta]$ with any $\delta>0$ we have $\lim _{m \rightarrow 1^{-}} \xi_{m} f=0$ and, by a corollary to the Banach-Steinhaus theorem, the above limit extends by density for any $f \in L^{1}[a, 1]$ and, in particular, for $f \equiv 1$ which yields (24).

Now, we observe that $\bar{\lambda}$, defined before (23), is universal for all $b^{a}$ satisfying $b^{a} \leq \bar{C} \mu$. Let us consider $b_{\mu}^{a}=\bar{C} \mu$. Then, for $f \geq 0$ and fixed $\lambda>\bar{\lambda}$, we have

$$
\left\|R\left(\lambda, A_{b_{\mu}^{a}}\right) f\right\|=\left\|R\left(\lambda, A_{0}\right) f\right\|+\frac{\left\langle b_{\mu}^{a}, R\left(\lambda, A_{0}\right) f\right\rangle}{1-\left\langle b_{\mu}^{a}, g^{-1} e_{-\lambda}\right\rangle} \int_{a}^{1} \frac{e_{-\lambda}(m)}{g(m)} d m=\int_{a}^{1} f(s) \Phi(s) d s
$$

where, by (20),

$$
\Phi(s)=e_{\lambda}(s) \int_{s}^{1} \frac{\left(1+C^{\prime} \mu(m)\right) e_{-\lambda}(m)}{g(m)} d m=C^{\prime}+\left(1-C^{\prime} \lambda\right) e_{\lambda}(s) \int_{s}^{1} \frac{e_{-\lambda}(m)}{g(m)} d m(25)
$$

with

$$
C^{\prime}=\frac{\bar{C}}{1-\left\langle b^{a}, g^{-1} e_{\lambda}\right\rangle} \int_{a}^{1} \frac{e_{-\lambda}(m)}{g(m)} d m .
$$

Now, $\Phi$ is a continuous function on $[a, 1]$ by (24) and it is strictly positive on $[a, 1)$ as both the integrand and $e_{\lambda}$ are positive on this interval. Moreover, if $s \rightarrow 1^{-}$then, again by (24), the rightmost term of (25) converges to zero and $\Phi(s) \rightarrow C^{\prime}>0$. Thus $\Phi(s) \geq C_{0}>0$ on $[a, 1]$, where $C_{0}$ is a constant and therefore $\left\|R\left(\lambda, A_{b_{\mu}^{a}}\right) f\right\| \geq C_{0}\|f\|$ for any $\lambda>\bar{\lambda}$. It is a classical result, see e.g. [14], that $A_{b_{\mu}^{a}}$ is densely defined. Thus, by the Arendt-Batty-Robinson theorem, [1, Theorem 2.5] or [4, Theorem 3.39], the operator $\left(A_{b_{\mu}^{a}}, D\left(A_{b_{\mu}^{a}}\right)\right)$ generates a positive semigroup. Since we are in an $L^{p}$ space, the type of the semigroup equals the spectral bound of $A_{b_{\mu}^{a}}$.

Now let us consider an arbitrary $b^{a}$ satisfying $b^{a} \leq \bar{C} \mu$. Then, by (23), for all $\lambda \geq \bar{\lambda}$, $0 \leq R\left(\lambda, A_{b^{a}}\right) \leq R\left(\lambda, A_{b_{\mu}^{a}}\right)$ and hence $0 \leq R^{n}\left(\lambda, A_{b^{a}}\right) \leq R^{n}\left(\lambda, A_{b_{\mu}^{a}}\right)$. Since $A_{b_{\mu}^{a}}$ generates a semigroup, its resolvent satisfies the Hille-Yosida estimates and hence also $\left\|R^{n}\left(\lambda, A_{b^{a}}\right)\right\| \leq$ $M(\lambda-\bar{\lambda})^{-n}$, for some $M>0$ and all $n \in \mathbb{N}$. Thus, $\left(A_{b^{a}}, D\left(A_{b^{a}}\right)\right)$ generates a positive semigroup.

In the last step we analyze the operator $P R\left(\lambda, A_{b^{a}}\right)$ and, more precisely, its spectral radius $r\left(P R\left(\lambda, A_{b^{a}}\right)\right)$. The key observation is that, by (14), the support of $P f$ is contained in $[a, 1-h]$ for any $f \in L^{1}[a, 1]$. Thus, by writing

$$
\left(P R\left(\lambda, A_{b^{a}}\right)\right)^{n} f=\left(P R\left(\lambda, A_{b^{a}}\right)\right)^{n-1} P R\left(\lambda, A_{b^{a}}\right) f,
$$

we can consider the powers $\left(P R\left(\lambda, A_{b^{a}}\right)\right)^{n} f$ with $n \geq 2$ as acting on $L^{1}[a, 1-h]$. Also, since we deal with positive operators, we can restrict ourselves to nonnegative functions. Hence, let $0 \leq v \in L^{1}[a, 1-h]$. Then we can write

$$
\left(R\left(\lambda, A_{0}\right) v\right)(m)= \begin{cases}\left(R\left(\lambda, A_{0}^{\prime}\right) v\right)(m) & \text { for } m \in[a, 1-h), \\ \frac{e_{-\lambda}(m)}{g(m)} L_{\lambda}(v) & \text { for } m \in[1-h, 1],\end{cases}
$$

where $A_{0}^{\prime}$ is the operator $A_{0}$ restricted to $[a, 1-h)$ and

$$
L_{\lambda}(v)=\int_{a}^{1-h} e_{\lambda}(s) v(s) d s \leq e_{\lambda}(1-h)\|v\|
$$


Hence

$$
\begin{aligned}
& \int_{a}^{1}\left(P R\left(\lambda, A_{0}\right) v\right)(m) d m \\
& \quad=\int_{a}^{1} b^{r}(m)\left(R\left(\lambda, A_{0}\right) v\right)(m) d m \\
& \quad=\int_{a}^{1-h} b^{r}(m)\left(R\left(\lambda, A_{0}^{\prime}\right) v\right)(m) d m+L_{\lambda}(v) \int_{1-h}^{1} \frac{b^{r}(m) e_{-\lambda}(m)}{g(m)} d m \\
& \quad \leq \frac{\sup _{m \in[a, 1-h]} b^{r}(m)\|v\|}{\lambda}+\|v\| \int_{1-h}^{1} \frac{b^{r}(m) e^{-\lambda(G(m)-G(1-h))-Q(m)+Q(1-h)}}{g(m)} d m,
\end{aligned}
$$

where we used the standard result that the transport problem with homogeneous Dirichlet boundary conditions and bounded coefficients generates a contractive semigroup. As for the second term, we note that $G(m)>G(1-h)$ for $m>1-h$ as $R$ is strictly increasing. Also, $b^{r} e_{-\lambda} / g \leq b^{r} e^{-Q} / g$ and the latter is integrable by (15) and (20). Thus, from the Lebesgue dominated convergence theorem, the integral converges to 0 as $\lambda \rightarrow \infty$ and we can write $\left\|P R\left(\lambda, A_{0}\right) v\right\| \leq c_{1}(\lambda)\|v\|$ for any $0 \leq v \in L^{1}[a, 1]$ with support in $[a, 1-h]$, where $c_{1}(\lambda) \rightarrow 0$ as $\lambda \rightarrow \infty$.

Next, for such functions $v$, again using (15) and argument as above, we find that

$$
\begin{aligned}
\int_{a}^{1} P\left(\frac{e_{-\lambda}}{g} \frac{\left\langle b^{a}, R\left(\lambda, A_{0}\right) v\right\rangle}{1-\left\langle b^{a}, g^{-1} e_{-\lambda}\right\rangle}\right)(m) d m & =\frac{\left\langle b^{a}, R\left(\lambda, A_{0}\right) v\right\rangle}{1-\left\langle b^{a}, g^{-1} e_{-\lambda}\right\rangle} \int_{a}^{1} \frac{b^{r}(m) e_{-\lambda}(m)}{g(m)} d m \\
& \leq \frac{c_{2}(\lambda)}{1-c_{3}(\lambda)} c_{3}(\lambda)\|v\|=c_{4}(\lambda)\|v\|,
\end{aligned}
$$

where $c_{3}(\lambda)$, and hence $c_{4}(\lambda)$, tend to 0 as $\lambda \rightarrow \infty$. Now, for arbitrary $f \in L^{1}[a, 1]$, for $r\left(P R\left(\lambda, A_{b^{a}}\right)\right)$ we take $\lambda>\bar{\lambda}$ and such that $c_{1}(\lambda)+c_{4}(\lambda)<1 / 2$. Then

$$
\left\|\left(P R\left(\lambda, A_{b^{a}}\right)\right)^{n} f\right\| \leq\left\|\left(P R\left(\lambda, A_{b^{a}}\right)\right)^{n-1}\right\|\left\|P R\left(\lambda, A_{b^{a}}\right)\right\|\|f\| \leq \frac{1}{2^{n-1}}\left\|P R\left(\lambda, A_{b^{a}}\right)\right\|\|f\|
$$

and

$$
r\left(P R\left(\lambda, A_{b^{a}}\right)\right)=\lim _{n \rightarrow \infty} \sqrt[n]{\left\|\left(P R\left(\lambda, A_{b^{a}}\right)\right)^{n}\right\|} \leq \frac{1}{2} .
$$

Hence, for sufficiently large $\lambda$ the resolvent $R\left(\lambda, A_{b^{a}}+P\right)$ is given by a norm converging series of positive operators and thus it is a positive operator. Therefore we can apply Desch's theorem, see e.g. [7] or [4, Theorem 5.13], to claim that $\left(A_{b^{a}}, D\left(A_{b^{a}}\right)\right)$ generates a positive semigroup.

Remark 2 Arguing as in (24) for $R\left(\lambda, A_{0}\right)$ and using $e_{-\lambda}(m) / g(m) \rightarrow 0$ as $m \rightarrow 1^{-}$we see from (23) that if $u \in D\left(A_{b^{a}}\right)$ then

$$
\lim _{m \rightarrow 1^{-}} u(m)=0 .
$$

\section{Reduction of $\{T(t)\}_{t \geq 0}$ to a Stochastic Semigroup}

Equation (7) can be rewritten as an evolution equation

$$
u^{\prime}(t)=B u(t)
$$


where

$$
B f(m)=g(m) f^{\prime}(m)-\mu(m) f(m)+\int_{a}^{1} f(y) \mathcal{P}(m, d y) .
$$

We recall that a positive semigroup is called stochastic if it is conservative on nonnegative initial data. The semigroup $\{T(t)\}_{t \geq 0}$ of Theorem 1 in general is not stochastic. However, using a suitable eigenvectors of the operator $B$, given by (28), we are able to construct a semigroup $\{U(t)\}_{t \geq 0}$ which is similar to $\{T(t)\}_{t \geq 0}$ and which is stochastic. This will facilitate analysis of stability of $\{T(t)\}_{t \geq 0}$.

We begin with the following proposition.

Proposition 1 The operator $B$ has an eigenvector $w$ which satisfies the following inequalities

$$
\alpha \leq w(m) \leq \beta \quad \text { for all } m \in[a, 1)
$$

where $0<\alpha<\beta$.

We divide the proof of Proposition 1 into a sequence of lemmata.

First observe that $B w_{\lambda}=\lambda w_{\lambda}$ if and only if $w_{\lambda}$ is a bounded function such that $w_{\lambda}$ is absolutely continuous in each interval $[a, 1-\varepsilon], \varepsilon>0$, and $w_{\lambda}$ satisfies the following equation

$$
w_{\lambda}^{\prime}(m)=d_{\lambda}(m) w_{\lambda}(m)-\frac{1}{g(m)} F w_{\lambda}(m)
$$

where

$$
d_{\lambda}(m)=\frac{\mu(m)+\lambda}{g(m)}, \quad \text { and } \quad F f(m)=\int_{a}^{1} f(y) \mathcal{P}(m, d y) .
$$

We note that due to (8) and the domain of definition of $P$, the function $F f$ is locally integrable on $[a, 1)$ for any $f \in L^{\infty}[a, 1]$. The following lemma gathers some properties of the operator $F$.

Lemma 1 Let $z \in[a+h, 1]$. If $w_{1}, w_{2} \in L^{\infty}[a, 1]$ and $w_{1}(m)=w_{2}(m)$ for $m \in[a, z-h]$, then $F w_{1}(m)=F w_{2}(m)$ for $m \in[a, z]$.

Proof Let $w \in L^{\infty}[a, 1]$. As $\mathcal{P}(m,(z-h, 1])=0$ for $m \in[a, z]$ we have

$$
F w(m)=\int_{a}^{z-h} w(y) \mathcal{P}(m, d y), \quad \text { for } m \leq z .
$$

Applying formula (31) to the function $w=w_{1}-w_{2}$ we get $F w_{1}(m)=F w_{2}(m)$ for $m \in$ $[a, z]$.

By Lemma 1, the restriction of the function $F w_{\lambda}$ to the interval $[a, z], z \in[a+h, 1]$, depends only on the restriction of $w_{\lambda}$ to the interval $[a, z-h]$. In particular $F w_{\lambda}(m)=0$ for $m \in[a, a+h]$. This implies that equation (29) takes the form

$$
w_{\lambda}^{\prime}(m)=d_{\lambda}(m) w_{\lambda}(m) \text { for } m \in[a, a+h] .
$$

If we assume that $w_{\lambda}(a)=1$, then equation (32) has a unique continuous solution. Since the function $F w_{\lambda}$, restricted to $[a+h, a+2 h]$, depends only on the function $w_{\lambda}$ restricted 
to $[a, a+h]$ and it is integrable on $[a, a+2 h]$, equation (29) has a unique solution in the interval $[a, a+2 h]$ such that $w_{\lambda}(a)=1$. This solution is an absolutely continuous function and depends continuously on $\lambda$. Using this method we can solve (29) successively for $m \in$ $[a+2 h, a+3 h], \ldots$. We summarize the above observation.

Lemma 2 For every $\lambda \in \mathbb{R}$ equation (29) has the unique solution $w_{\lambda}:[a, 1) \rightarrow \mathbb{R}$ such that $w_{\lambda}(a)=1$. The solution $w_{\lambda}$ is an absolutely continuous function in each interval $[a, 1-$ $\varepsilon], \varepsilon>0$. This solution depends continuously on $\lambda$, i.e. for every $\varepsilon>0$ the map $\varphi: \mathbb{R} \rightarrow$ $C[a, 1-\varepsilon]$ given by $\varphi(\lambda)=w_{\lambda}$ is continuous.

Lemma 3 There exists $\lambda_{1}>0$ such that for every $\lambda \geq \lambda_{1}$ the function $w_{\lambda}$ is increasing.

Proof Let $\lambda_{1}$ be a constant such that

$$
\lambda_{1}>b(m)+\frac{\bar{C}}{h} g(m) \text { for } m \leq 1-\frac{h}{2} .
$$

Since $b(m)=0$ for $m \leq a+h$, the function $w_{\lambda}$ satisfies the equation $w_{\lambda}^{\prime}=d_{\lambda} w_{\lambda}$ in the interval $[a, a+h]$. Further, $d_{\lambda}>0$ implies that $w_{\lambda}$ is increasing in $[a, a+h]$. Now, we assume that $w_{\lambda}$ is increasing in $\left[a, m_{0}\right]$, where $m_{0} \leq 1-\frac{3}{2} h$. We claim that $w_{\lambda}$ is increasing in the interval $\left[a, m_{0}+h\right]$. Indeed, from (31) we have

$$
F w_{\lambda}(m) \leq b(m) \max \left\{w_{\lambda}(x): x \in[a, m-h]\right\} \leq b(m) w_{\lambda}\left(m_{0}\right)
$$

for $m \in\left[m_{0}, m_{0}+h\right]$. Thus

$$
w_{\lambda}^{\prime}(m)=d_{\lambda}(m) w_{\lambda}(m)-\frac{1}{g(m)} F w_{\lambda}(m) \geq \frac{1}{g(m)}\left(\lambda w_{\lambda}(m)-b(m) w_{\lambda}\left(m_{0}\right)\right)
$$

for $m \in\left[m_{0}, m_{0}+h\right]$. Since $\lambda>b(m)$ for $m \leq 1-\frac{h}{2}$ and since the function $w_{\lambda}$ is locally absolutely continuous inequality, (34) implies that $w_{\lambda}$ is increasing in the interval $\left[m_{0}, m_{0}+h\right]$. Hence, the function $w_{\lambda}$ is increasing in the interval $\left[a, 1-\frac{h}{2}\right]$. Moreover, (33) and the inequality $F w_{\lambda}(m) \leq b(m) w_{\lambda}(m)$ for $m \leq 1-\frac{h}{2}$ imply that $w_{\lambda}^{\prime}(m) \geq \frac{\bar{C}}{h} w_{\lambda}(m)$ and, consequently,

$$
w_{\lambda}\left(1-\frac{h}{2}\right) \geq e^{\frac{\bar{C}}{h} \frac{h}{2}} w_{\lambda}(1-h)>\bar{C} w_{\lambda}(1-h)
$$

From (35) we obtain

$$
F w_{\lambda}(m) \leq b(m) w_{\lambda}(1-h)<\bar{C}^{-1} b(m) w_{\lambda}\left(1-\frac{h}{2}\right) \leq \mu(m) w_{\lambda}\left(1-\frac{h}{2}\right)
$$

for $m \in[1-h, 1]$. Since $d_{\lambda}(m)>\frac{\mu(m)}{g(m)}$, inequality (36) implies that

$$
w_{\lambda}^{\prime}(m)=d_{\lambda}(m) w_{\lambda}(m)-\frac{1}{g(m)} F w_{\lambda}(m)>d_{\lambda}(m)\left[w_{\lambda}(m)-w_{\lambda}\left(1-\frac{h}{2}\right)\right] .
$$

Writing this inequality as $\left[w_{\lambda}(m)-w_{\lambda}(1-h / 2)\right]^{\prime}>d_{\lambda}(m)\left[w_{\lambda}(m)-w_{\lambda}(1-h / 2)\right]$, we find $w_{\lambda}(m)>w_{\lambda}(1-h / 2)$ for $m \geq h / 2$ and consequently the function $w_{\lambda}$ is increasing in $\left[1-\frac{h}{2}, 1\right)$.

Lemma 4 There exists $\lambda_{2}$ such that for each $\lambda \leq \lambda_{2}$ the function $w_{\lambda}$ has a zero in the interval $(a, 1)$. 
Proof Let $\tilde{m} \in(a+h, 1)$ be a point such that $b(\tilde{m})>0$. Since $b$ is a continuous function, there exist positive constants $\varepsilon, \delta$ such that $\delta<h, \widetilde{m}+\delta<1$ and

$$
b(m) \geq \varepsilon \quad \text { for } m \in[\tilde{m}-\delta, \tilde{m}+\delta] .
$$

Let us denote $\alpha_{\lambda}=\max \left\{d_{\lambda}(m): m \leq \tilde{m}+\delta\right\}$ and choose $\lambda_{2}$ such that for every $\lambda \leq \lambda_{2}$ we have $\alpha_{\lambda}<0$ and

$$
\varepsilon \delta e^{-\alpha_{\lambda}(h-\delta)}>g(m) \quad \text { for } m \leq \tilde{m}+\delta .
$$

We prove that the function $w_{\lambda}$ has a zero in the interval $[a, \tilde{m}+\delta]$. Conversely, suppose that $w_{\lambda}(m)>0$ in the interval $[a, \tilde{m}+\delta]$. Since $w_{\lambda}^{\prime}(m) \leq \alpha_{\lambda} w_{\lambda}(m)$ for $m \leq \tilde{m}+\delta$, the function $w_{\lambda}$ is decreasing in the interval $[a, \tilde{m}+\delta]$ and

$$
w_{\lambda}(m) \geq e^{-\alpha_{\lambda}(\tilde{m}-m)} w_{\lambda}(\tilde{m}) \quad \text { for } m \leq \tilde{m} .
$$

From inequality (37) it follows that

$$
F w_{\lambda}(m) \geq \varepsilon \inf \left\{w_{\lambda}(x): x \leq m-h\right\}=\varepsilon w_{\lambda}(m-h)
$$

for $m \in[\tilde{m}-\delta, \tilde{m}+\delta]$. From (39) and (40) it follows that

$$
F w_{\lambda}(m) \geq \varepsilon e^{-\alpha_{\lambda}(\tilde{m}-m+h)} w_{\lambda}(\tilde{m}) \geq \varepsilon e^{-\alpha_{\lambda}(h-\delta)} w_{\lambda}(\tilde{m}) .
$$

Inequalities (38), (41) and $w_{\lambda}^{\prime}(m) \leq-\frac{1}{g(m)} F w_{\lambda}(m)$ imply that

$$
w_{\lambda}^{\prime}(m) \leq-\varepsilon e^{-\alpha_{\lambda}(h-\delta)} \frac{1}{g(m)} w_{\lambda}(\tilde{m})<-\frac{1}{\delta} w_{\lambda}(\tilde{m}) .
$$

Thus

$$
w_{\lambda}(\tilde{m}+\delta)<w_{\lambda}(\tilde{m})-\delta\left(\frac{1}{\delta} w_{\lambda}(\tilde{m})\right)=0
$$

and therefore the function $w_{\lambda}$ has a zero in the interval $[a, \tilde{m}+\delta]$.

Now we denote by $\Lambda$ the subset of $\mathbb{R}$ which contains all constants $\lambda \in \mathbb{R}$ such that $w_{\lambda}\left(m_{\lambda}\right)=0$ for some $m_{\lambda} \in(a, 1)$. According to Lemma 4 the set $\Lambda$ is nonempty and, by Lemma 3 , it is bounded from above. Let $\lambda_{s}=\sup \Lambda$.

Lemma 5 There exists $\alpha>0$ such that $w_{\lambda_{s}}(m) \geq \alpha$ for $m \in[a, 1)$.

Proof The solution $w_{\lambda}$ depends continuously on $\lambda$. From this and from the definition of $\lambda_{s}$, it follows that $w_{\lambda_{s}}(m) \geq 0$ for $m \in[a, 1)$. We claim that $w_{\lambda_{s}}(m)>0$ for $m \in[a, 1)$. Suppose, contrary to our claim, that there exists $m_{s} \in(a, 1)$ such that $w_{\lambda_{s}}\left(m_{s}\right)=0$ and $w_{\lambda_{s}}(m)>0$ for $m \in\left[a, m_{s}\right)$. Since $w_{\lambda_{s}} \geq 0$, we have $F w_{\lambda_{s}} \geq 0$. Thus the function $w_{\lambda_{s}}$ satisfies the inequality $w_{\lambda_{s}}^{\prime} \leq d_{\lambda_{s}} w_{\lambda_{s}}$ and the condition $w_{\lambda_{s}}\left(m_{s}\right)=0$. By standard arguments from the theory of differential inequalities we obtain $w_{\lambda_{s}}(m) \leq 0$ for $m \geq m_{s}$. On the other hand, the function $w_{\lambda_{s}}$ is nonnegative. Thus $w_{\lambda_{s}}(m)=0$ for $m \geq m_{s}$ and from (29) we have $F w_{\lambda_{s}}(m)=0$ for $m \geq m_{s}$. But since $w_{\lambda_{s}}(m)>0$ for $m \in\left[a, m_{s}\right)$, inequality (12) implies that $\int_{m_{s}}^{1} F w_{\lambda_{s}}(m) d m>0$, which contradicts the fact that $F w_{\lambda_{s}}(m)=0$ for $m \in\left[m_{s}, 1\right)$. Thus $w_{\lambda_{s}}(m)>0$ for $m \in[a, 1)$. Let $\varepsilon=\min _{m \leq 1-h} w_{\lambda_{s}}(m)$. Since $\mathcal{P}_{k}(m,[a, 1-h])=1$ and $w_{\lambda_{s}} \geq \varepsilon \mathbf{1}_{[a, 1-h]}$ we have, using also (31),

$$
F w_{\lambda_{s}}(m) \geq \varepsilon F \mathbf{1}_{[a, 1-h]}(m)=\varepsilon \sum_{k=1}^{\infty} k b_{k}(m) \mathcal{P}_{k}(m,[a, 1-h])=\varepsilon b(m), \quad m \in[a, 1) .
$$


This implies that for all $m \in(a, 1)$

$$
w_{\lambda_{s}}^{\prime}(m) \leq d_{\lambda_{s}}(m) w_{\lambda_{s}}(m)-\varepsilon j(m),
$$

where $j(m)=b(m) / g(m)$. From (15) it follows that there exists a constant $\gamma>1$ such that $d_{\lambda_{s}}(m) \leq \gamma j(m)$ for $m \geq 1-h$. We check that $w_{\lambda_{s}} \geq \alpha$, where $\alpha=\varepsilon / 2 \gamma$. Conversely, suppose that for some $m_{0} \in(1-h, 1)$ we have $w_{\lambda_{s}}\left(m_{0}\right)<\alpha$. Then $w_{\lambda_{s}}(m)<\alpha$ for $m \in$ $\left[m_{0}, 1\right)$. Indeed, if there exists $m_{1}>m_{0}$ such that $w_{\lambda_{s}}\left(m_{1}\right)=\alpha$ and $w_{\lambda_{s}}(m) \leq \alpha$ for $m \in$ $\left[m_{0}, m_{1}\right]$, then from

$$
w_{\lambda_{s}}^{\prime}(m) \leq d_{\lambda_{s}}(m) w_{\lambda_{s}}(m)-\varepsilon j(m)<-\frac{\varepsilon}{2} j(m)<0, \quad m \in\left[m_{0}, m_{1}\right],
$$

it follows that $w_{\lambda_{s}}(m)<\alpha$ for $m \in\left[m_{0}, m_{1}\right]$, which contradicts the definition of $m_{1}$. Thus $w_{\lambda_{s}}(m)<\alpha$ for $m \in\left[m_{0}, 1\right)$. From (46) it follows that

$$
w_{\lambda_{s}}(m)-w_{\lambda_{s}}\left(m_{0}\right)=\int_{m_{0}}^{m} w_{\lambda_{s}}^{\prime}(x) d x \leq-\frac{\varepsilon}{2} \int_{m_{0}}^{m} j(x) d x .
$$

However, since $\int_{m_{0}}^{1} j(x) d x=\infty$, (47) yields $\lim _{m \rightarrow 1} w_{\lambda_{s}}(m)=-\infty$, which contradicts the fact that $w_{\lambda_{s}} \geq 0$ and completes the proof that $w_{\lambda_{s}} \geq \alpha$.

Lemma 6 There exists $\beta>0$ such that $w_{\lambda_{s}}(m) \leq \beta$ for $m \in[a, 1)$.

Proof Now we show that there exists $\beta>0$ such that $w_{\lambda_{s}} \leq \beta$. Let $M>0$ be such that $w_{\lambda_{s}}(m)<M$ for $m \in[a, 1-h]$. Let

$$
\gamma=\max _{m \leq 1} \frac{\left|\lambda_{s}\right|+1}{g(m)} \text { and } \beta=\bar{C} e^{\gamma} M .
$$

We claim that $w_{\lambda_{s}} \leq \beta$. Suppose to the contrary that there exists $\bar{m} \in(1-h, 1)$ such that $w_{\lambda_{s}}(\bar{m})>\beta$. From the continuous dependence of the functions $w_{\lambda}$ on $\lambda$ it follows that there exists $\lambda \in \Lambda$ such that $\left|\lambda-\lambda_{s}\right|<1, w_{\lambda}(\bar{m})>\beta, w_{\lambda}(m)>0$ for $m \in[a, \bar{m}]$, and $w_{\lambda}(m)<M$ for $m \in[a, 1-h]$. According to (31) we have

$$
F w_{\lambda}(m) \leq M b(m) \text { for } m \in[a, 1] .
$$

Since

$$
d_{\lambda}(m)=\frac{\lambda+\mu(m)}{g(m)} \geq-\gamma+\frac{\mu(m)}{g(m)} \geq-\gamma+\bar{C}^{-1} j(m),
$$

the function $w_{\lambda}$ satisfies the inequality

$$
w_{\lambda}^{\prime}(m) \geq\left(-\gamma+\bar{C}^{-1} j(m)\right) w_{\lambda}(m)-M j(m) \geq-\gamma w_{\lambda}(m)
$$

provided that $w_{\lambda}(m) \geq \bar{C} M$. However, $w_{\lambda}(\bar{m})>\beta=\bar{C} e^{\gamma} M>\bar{C} M$ hence $w_{\lambda} \geq \bar{C} M$ in some neighborhood of $\bar{m}$. Then, in this neighborhood, (51) yields

$$
w_{\lambda}(m) \geq e^{-\gamma(m-\bar{m})} w_{\lambda}(\bar{m})>e^{-\gamma} \beta=\bar{C} M
$$

and, due to the strict inequality, we can extend (51) to a larger domain. Consequently, $w_{\lambda}(m)>\bar{C} M$ for $m \in[\bar{m}, 1)$ and hence $w_{\lambda}(m)>0$ for all $m \in[a, 1)$, which is impossible because $\lambda \in \Lambda$ and the function $w_{\lambda}$ has a zero in the interval $[a, 1)$. This implies that $w_{\lambda_{s}} \leq \beta$.

Now, denote by $M_{w}$ the operator of multiplication by the solution $w$ of (29) for an appropriate $\lambda \in \mathbb{R}$. Since $w$ is an absolutely continuous function $w:[a, 1) \rightarrow(0, \infty)$ satisfying 
$\alpha \leq w(m) \leq \beta$ for all $m \in[a, 1)$, where $0<\alpha<\beta, M_{w}$ is an isomorphism of $L^{1}[a, 1]$. Defining

$$
U(t)=e^{-\lambda t} M_{w} T(t) M_{w^{-1}},
$$

we see that $\{U(t)\}_{t \geq 0}$ is a strongly continuous positive semigroup on $L^{1}[a, 1]$ with the generator

$$
\begin{aligned}
\mathcal{A} u & =M_{w}\left(A_{b^{a}}+P\right) M_{w^{-1}} u-\lambda u=\frac{w^{\prime} g u}{w}-(g u)^{\prime}-\mu u+w P\left(\frac{u}{w}\right)-\lambda u \\
& =-(g u)^{\prime}-\frac{u}{w} \int_{a}^{1} w(y) \mathcal{P}(\cdot, d y)+w P\left(\frac{u}{w}\right)
\end{aligned}
$$

on the domain $D(\mathcal{A})=M_{w^{-1}} D\left(A_{b^{a}}\right)$. In particular, for $u \in D(\mathcal{A})$

$$
g(a) u(a)=\int_{a}^{1} \frac{b^{a}(m) w(a)}{w(m)} u(m) d m .
$$

Since for $u \in D(\mathcal{A})$ the last two terms in the definition of $\mathcal{A}$ are in $L^{1}[a, 1]$ (as $u / w \in$ $\left.L_{b}^{1}[a, 1]\right)$, also $(g u)^{\prime} \in L^{1}[a, 1]$ and we can integrate termwise to get

$$
\begin{aligned}
& \int_{a}^{1}(\mathcal{A} u)(m) d m \\
&= g(a) u(a)-\lim _{m \rightarrow 1^{-}} g(m) u(m) \\
& \quad-\int_{a}^{1} \frac{u(m)}{w(m)}\left(w(a) b^{a}(m)+\int_{a}^{1} w(y) \mathcal{P}^{r}(m, d y)\right) d m \\
&+\int_{a}^{1} w(m)\left(P\left(\frac{u}{w}\right)\right)(m) d m \\
&=-\int_{a}^{1} \frac{u(m)}{w(m)} \int_{a}^{1} w(y) \mathcal{P}^{r}(m, d y) d m+\int_{a}^{1} w(m)\left(P\left(\frac{u}{w}\right)\right)(m) d m=0,
\end{aligned}
$$

where we used in the following order, (26), definition of $b^{a}$ and $\mathcal{P}^{r},(55)$ and the definition of the operator $P$. Thus, we have proved

Proposition $2\{U(t)\}_{t \geq 0}$ is a stochastic semigroup on $L^{1}[a, 1]$.

\section{Invariant Density}

We need some auxiliary definitions. Denote by $D$ the set of all densities i.e., the set defined by

$$
D=\left\{f \in L^{1}[a, 1]: f \geq 0, \int_{a}^{1} f(m) d m=1\right\} .
$$

A density $f_{*}$ is called invariant under a stochastic semigroup $\{U(t)\}_{t \geq 0}$ if $U(t) f_{*}=f_{*}$ for every $t \geq 0$.

Now we will investigate the existence of an invariant density of the semigroup $\{U(t)\}_{t \geq 0}$. Before, however, we introduce some simplifying notation. Let $\tilde{b}(m)=b^{a}(m) w(a) / w(m)$ and $R f=w P\left(\frac{f}{w}\right)$. Then, using (8), we can write $\frac{1}{w} P^{*} w=R^{*} \mathbf{1}$ 
Since $R f=w P\left(\frac{f}{w}\right)$ and $0<\alpha \leq w(m) \leq \beta$ for $m \in[a, 1]$, we have

$$
\frac{\alpha}{\beta} P f \leq R f \leq \frac{\beta}{\alpha} P f
$$

for $f \geq 0, f \in L_{b}^{1}[a, 1]$. We also have

$$
\frac{\alpha}{\beta} b^{r}(m) \leq R^{*} \mathbf{1}(m) \leq \frac{\beta}{\alpha} b^{r}(m) \quad \text { and } \quad \frac{\alpha}{\beta} b^{a}(m) \leq \tilde{b}(m) \leq \frac{\beta}{\alpha} b^{a}(m)
$$

for $m \in[a, 1]$.

Proposition 3 The semigroup $\{U(t)\}_{t \geq 0}$ has a unique invariant density $v_{*} \in D(\mathcal{A})$. Additionally, $v_{*}>0$ on $(a, 1)$ and $v_{*}(1)=0$.

Proof An invariant density $v_{*}$ of the semigroup $\{U(t)\}_{t \geq 0}$ is a solution of the following stationary system

$$
\begin{aligned}
(g(m) v(m))^{\prime} & =-\tilde{b}(m) v(m)-R^{*} \mathbf{1}(m) v(m)+R v(m), \\
g(a) v(a) & =\int_{a}^{1} \tilde{b}(m) v(m) d m .
\end{aligned}
$$

We check that the system (58)-(59) has a unique solution such that $v(1-h)=1$. First, since $R v(m)=0$ for $m \geq 1-h$, in the interval $[1-h, 1]$ the function $v$ satisfies the differential equation $v^{\prime}(m)=-\gamma(m) v(m)$, where $\gamma(m)=\left(g^{\prime}(m)+\tilde{b}(m)+R^{*} \mathbf{1}(m)\right) / g(m)$. Hence

$$
v(m)=\exp \left\{-\int_{1-h}^{m} \gamma(y) d y\right\}
$$

for $m \geq 1-h$. Arguing as in the proof of Theorem 1 , by (57), $\lim _{m \rightarrow 1^{-}} v(m)=0$ and $\int_{1-h}^{1} b(m) v(m) d m<\infty$. Since, as the operator $P, R v(m)$ only depends on values of $v$ on $[m+h, 1]$, the last inequality implies that $R v(m)$ is a well defined and integrable function on $[1-2 h, 1-h]$. Thus we can solve equation (58) on the interval $[1-2 h, 1-h]$ matching the value $v(1-h)$ and step by step we construct an absolutely continuous solution of this equation on the interval $[a, 1)$. By (57) and the integrability of $b v$, all terms on the right hand side of (58) are integrable over the interval $[a, 1]$. Hence, integrating (58) termwise, we obtain

$$
g(1) v(1)-g(a) v(a)=-\int_{a}^{1} \tilde{b}(m) v(m) d m-\int_{a}^{1} R^{*} \mathbf{1}(m) v(m) d m+\int_{a}^{1} R v(m) d m .
$$

Since $v(1)=0$ and $\int_{a}^{1} R^{*} \mathbf{1}(m) v(m) d m=\int_{a}^{1} R v(m) d m$, we get

$$
g(a) v(a)=\int_{a}^{1} \tilde{b}(m) v(m) d m .
$$

From (58) we see that $(g v)^{\prime} \in L^{1}[a, 1]$. Denoting $u=w v$, where $w$ was used to define $\{U(t)\}_{t \geq 0}$, we have

$$
(g u)^{\prime}=(g v w)^{\prime}=w(g v)^{\prime}+g v w^{\prime}=w(g v)^{\prime}+g v \mu\left(w^{\prime} / \mu\right) .
$$

By construction and (15), $v \mu$ is integrable and $w / \mu$ is bounded, by (29) and again by (15). The fact that $u$ satisfies other requirements for belonging to $D\left(A_{b^{a}}\right)$ is clear. Therefore $v \in D(\mathcal{A})$. Now we check that $v(m)>0$ for $m \in(a, 1)$. Suppose to the contrary that for 
some $m \in(a, 1) v(m)=0$ and $v(y)>0$ for $y \in(m, 1)$. Then, integrating both sides of (58) over the interval $[a, m]$, we obtain

$$
g(m) v(m)-g(a) v(a)=-\int_{a}^{m} \tilde{b}(y) v(y) d y-\int_{a}^{m} R^{*} \mathbf{1}(y) v(y) d y+\int_{a}^{m} R v(y) d y .
$$

From the boundary condition (59), it follows that

$$
\int_{m}^{1} \tilde{b}(y) v(y) d y-\int_{a}^{m} R^{*} \mathbf{1}(y) v(y) d y+\int_{a}^{1} R^{*} \mathbf{1}_{[a, m]}(y) v(y) d y=0 .
$$

Let $\overline{\mathcal{P}}(m, d y)=\frac{w(y)}{w(m)} \mathcal{P}^{r}(m, d y)$. Then $R^{*} f(m)=\int_{a}^{1} f(y) \overline{\mathcal{P}}(m, d y)$ and we have

$$
\int_{m}^{1} \tilde{b}(y) v(y) d y-\int_{a}^{m} \overline{\mathcal{P}}(y,[a, 1]) v(y) d y+\int_{a}^{1} \overline{\mathcal{P}}(y,[a, m]) v(y) d y=0,
$$

but, since $\overline{\mathcal{P}}(y,[m, 1])=0$ for $y \leq m$, we obtain

$$
\int_{m}^{1} \tilde{b}(y) v(y) d y+\int_{m}^{1} \overline{\mathcal{P}}(y,[a, m]) v(y) d y=0 .
$$

As $v(y)>0$ for $y>m$, we have $\tilde{b}(y)=0$ and $\overline{\mathcal{P}}(y,[a, m])=0$ for almost every $y \geq m$. It means that $\mathcal{P}(y,\{a\})=b^{a}(y)=0$ and $\mathcal{P}^{r}(y,[a, m])=0$ for almost every $y \geq m$, which contradicts (12). Finally, we set $v_{*}=v /\|v\|$ and $v^{*}$ is a unique invariant density of the semigroup $\{U(t)\}_{t \geq 0}$.

\section{Proof of Theorem 2}

We precede the proof of Theorem 2 with some remarks. Let $\{U(t)\}_{t \geq 0}$ be the stochastic semigroup defined in Proposition 2. The semigroup $\{U(t)\}_{t \geq 0}$ is called asymptotically stable if there is an invariant density $v_{*}$ such that

$$
\lim _{t \rightarrow \infty}\left\|U(t) v-v_{*}\right\|=0 \quad \text { for } v \in D .
$$

Observe that if $\{U(t)\}_{t \geq 0}$ is asymptotically stable, then for every function $v \in L^{1}[a, 1]$ we have

$$
\lim _{t \rightarrow \infty} U(t) v=v_{*} \int_{a}^{1} v(m) d m \quad \text { in } L^{1}[a, 1]
$$

Since

$$
e^{-\lambda t} N(t, m)=\frac{u(t, m)}{w(m)} \quad \text { and } \quad \int_{a}^{1} u(0, m) d m=\int_{a}^{1} N(0, m) w(m) d m
$$

we have

$$
e^{-\lambda t} N(t, \cdot) \rightarrow \frac{v_{*}}{w} \int_{a}^{1} N(0, m) w(m) d m \quad \text { in } L^{1}[a, 1] .
$$

Thus it remains to check that under the assumptions of Theorem 2, the semigroup $\{U(t)\}_{t \geq 0}$ is asymptotically stable. It means that it is enough to show asymptotic stability of the semigroup $\{U(t)\}_{t \geq 0}$ if one of the conditions (I), (II) or (III) of Theorem 2 holds.

Proposition 4 Assume that one of the conditions (I), (I) or (III) holds. Then the semigroup $\{U(t)\}_{t \geq 0}$, defined in Proposition 2, is asymptotically stable. 
We precede the proof of Proposition 4 by an auxiliary definition. Let $\{U(t)\}_{t \geq 0}$ be a semigroup on the space $L^{p}(X, \Sigma, v), 1 \leq p \leq \infty$. We say that this semigroup $\{U(t)\}_{t \geq 0}$ is partially integral if there is $t_{0}>0$ such that the operator $U\left(t_{0}\right)$ can be written in the form

$$
U\left(t_{0}\right) f(m)=\int k(m, y) f(y) v(d y)+S f(m),
$$

where $S$ is a positive operator on $L^{p}$ and the kernel $k$ is a measurable nonnegative function such that

$$
\iint k(m, y) v(d y) v(d m)>0
$$

In order to prove Proposition 4 we need the following criterion for asymptotic stability of stochastic semigroups.

Proposition 5 ([25]) Let $\{U(t)\}_{t \geq 0}$ be a partially integral stochastic semigroup on the space $L^{1}(X, \Sigma, v)$. Assume that the semigroup $\{U(t)\}_{t \geq 0}$ has a unique invariant density $f_{*}$. If $f_{*}>0$ then the semigroup $\{U(t)\}_{t \geq 0}$ is asymptotically stable.

It remains to prove the following lemma.

Lemma 7 Assume that one of the conditions (I), (II) or (III) holds. Then the semigroup $\{U(t)\}_{t \geq 0}$ defined in Proposition 2 is partially integral.

Proof Let $\{S(t)\}_{t \geq 0}$ be the strongly continuous semigroup on $L^{1}[a, 1]$ generated by $\left(A_{b^{a}}, D\left(A_{b^{a}}\right)\right)$. Since the semigroup $\{T(t)\}_{t \geq 0}$ generated by $A_{b^{a}}+P$ can be considered as the Miyadera-Voigt perturbation of $\{S(t)\}_{t \geq 0}$, see [4, Lemma 5.14],

$$
T(t) f=S(t) f+\int_{0}^{t} T(t-\tau) P S(\tau) f d \tau, \quad f \in L^{1}[a, 1],
$$

which implies, in particular,

$$
T(t) f \geq S(t) f+\int_{0}^{t} S(t-\tau) P S(\tau) f d \tau, \quad 0 \leq f \in L^{1}[a, 1] .
$$

According to [9, Chap. V, Lemma B (i)], if $R$ is a positive operator and $Q$ is a positive and integral operator, then the operators $R Q$ and $Q R$ are integral operators. Therefore, if the semigroup $\{S(t)\}_{t \geq 0}$ is partially integral or the operator $P$ is partially integral, then from (65) it follows that the semigroup $\{T(t)\}_{t \geq 0}$ is partially integral.

If condition (I) holds, then

$$
P f(m) \geq \int_{a}^{1} q(m, y) f(y) d y
$$

for $f \geq 0$. Thus, the operator $P$ is partially integral which implies that the semigroup $\{T(t)\}_{t \geq 0}$ is partially integral.

If condition (II) holds, then $b^{a}(m)>0$ on some nontrivial interval. We check that the semigroup $\{S(t)\}_{t \geq 0}$ is partially integral, and consequently, the semigroup $\{T(t)\}_{t \geq 0}$ is partially integral. In this case there exist $m_{0} \in(a, 1), \varepsilon>0$ such that $b^{a}(m)>0$ for $m \in\left(m_{0}-\varepsilon, m_{0}+\varepsilon\right)$. Without loss of generality we can assume that $m_{0}+\varepsilon<1$. Let us denote by $\pi_{t} m_{0}$ the solution $m(t)$ of the equation $m^{\prime}=g(m)$ with the initial condition $m(0)=m_{0}$. Fix $t>0$ such that $\pi_{-t} m_{0} \geq m_{0}-\varepsilon$. Let $u$ be the solution of the equation $u^{\prime}(t)=A_{b^{a}} u$ with the initial condition $u(0, m)=v(m)$. Then there exists $\gamma_{1}>0$ such that 
$u(s, y) \geq \gamma_{1} v\left(\pi_{-s} y\right)$ for $y \in\left(m_{0}, m_{0}+\varepsilon\right)$ and $s \leq t$. Let $m \leq \pi_{t} a$. Then there exists $\gamma_{2}>0$ such that $u(t, m)>\gamma_{2} u(t-\tau(m), a)$, where $\tau(m)$ is given by the formula $\pi_{\tau(m)} a=m$. From the boundary condition (10) we obtain

$$
\begin{aligned}
u(t, m) & \geq \gamma_{2} u(t-\tau(m), a) \geq \frac{\gamma_{2}}{g(a)} \int_{a}^{1} b^{a}(y) u(t-\tau(m), y) d y \\
& \geq \frac{\gamma_{1} \gamma_{2}}{g(a)} \int_{m_{0}}^{m_{0}+\varepsilon} b^{a}(y) v\left(\pi_{\tau(m)-t} y\right) d y \\
& \geq \frac{\gamma_{1} \gamma_{2}}{g(a)} \int_{\pi_{\tau(m)-t} m_{0}}^{\pi_{\tau(m)-t}\left(m_{0}+\varepsilon\right)} \frac{b^{a}\left(\pi_{t-\tau(m)} x\right) g\left(\pi_{t-\tau(m)} x\right)}{g(x)} v(x) d x
\end{aligned}
$$

for $m \leq \pi_{t} a$. Thus $S(t) v(m) \geq \int k(x, m) v(x) d x$, where $k$ is a positive function for $m \in$ $\left(a, \pi_{t} a\right)$ and $x \in\left(\pi_{\tau(m)-t} m_{0}, \pi_{\tau(m)-t}\left(m_{0}+\varepsilon\right)\right)$ and the operator $S(t)$ is partially integral.

Now assume that condition (III) holds. Let $f \in L^{1}[a, 1]$ be a nonnegative function. We check that for small $t$ the operator $T(t)$ is partially integral, which is equivalent to the fact that the conjugated operator $T^{*}(t)$ has a nontrivial integral part. We have

$$
T^{*}(t) f(m) \geq \int_{0}^{t}\left[S^{*}(\tau) P^{*} S^{*}(t-\tau) f\right](m) d t \geq \varepsilon \int_{0}^{t} f\left(\pi_{t-\tau} r\left(\pi_{\tau} m\right)\right) d \tau,
$$

where $\varepsilon$ is a positive constant. Let $y(\tau, m)=\pi_{t-\tau} r\left(\pi_{\tau} m\right)$. Since

$$
\frac{\partial y}{\partial \tau}\left(0, m_{0}\right)=-g\left(r\left(m_{0}\right)\right)+r^{\prime}\left(m_{0}\right) g\left(m_{0}\right) \neq 0
$$

we have also $\frac{\partial y}{\partial \tau}(\tau, m) \neq 0$ for small $t, \tau \in[0, t]$ and $m \in\left(m_{0}-\delta, m_{0}+\delta\right)$. From (67) it follows that there are constants $\eta>0, \delta^{\prime}>0, s_{1} \in[a, 1], s_{2} \in[a, 1], s_{2}>s_{1}$ and $t_{0}>0$ such that

$$
T^{*}\left(t_{0}\right) f(m) \geq \eta \int_{s_{1}}^{s_{2}} f(y) d y
$$

for $m \in\left(m_{0}-\delta^{\prime}, m_{0}+\delta^{\prime}\right)$. From (68) it follows that the semigroup $\{T(t)\}_{t \geq 0}$ is partially integral.

Since $\{U(t)\}_{t \geq 0}$ is given by (53), it is also partially integral.

Acknowledgements This research was partially supported by the State Committee for Scientific Research (Poland) Grants No. N N201 608240 and N N201 605640, by EU FP6 Marie Curie RTN programme CODY and the International Sciences and Technology Agreements: Poland-SA Bilateral Agreement (GUN 2075457) and the University of KwaZulu-Natal Research Fund.

Open Access This article is distributed under the terms of the Creative Commons Attribution Noncommercial License which permits any noncommercial use, distribution, and reproduction in any medium, provided the original author(s) and source are credited.

\section{References}

1. Arendt, W.: Resolvent positive operators. Proc. Lond. Math. Soc. 54(3), 321-349 (1987)

2. Arino, O., Kimmel, M.: Comparison of approaches to modeling of cell population dynamics. SIAM J. Appl. Math. 53, 1480-1504 (1993)

3. Arino, O., Sanchez, E., Webb, G.F.: Polynomial growth dynamics of telomere loss in a heterogeneous cell population. Dyn. Contin. Discrete Impuls. Syst. 3, 263-282 (1997)

4. Banasiak, J., Arlotti, L.: Perturbations of Positive Semigroups with Applications. Springer, London (2006) 
5. Banasiak, J., Lamb, W.: Coagulation, fragmentation and growth processes in a size structured population. Discrete Contin. Dyn. Syst., Ser. B 11(3), 563-585 (2009)

6. Bell, G.I., Anderson, E.C.: Cell growth and division. I. A mathematical model with applications to cell volume distributions in mammalian suspension cultures. Biophys. J. 7, 329-351 (1967)

7. Desch, W.: Perturbations of positive semigroups in AL-spaces (1988), preprint

8. Diekmann, O., Heijmans, H.J.A.M., Thieme, H.R.: On the stability of the cell size distribution. J. Math. Biol. 19, 227-248 (1984)

9. Foguel, S.R.: The Ergodic Theory of Markov Processes. Van Nostrand Reinhold Comp., New York (1969)

10. Gyllenberg, M., Heijmans, H.J.A.M.: An abstract delay-differential equation modelling size dependent cell growth and division. SIAM J. Math. Anal. 18, 74-88 (1987)

11. Gyllenberg, M., Webb, G.F.: Age-size structure in populations with quiescence. Math. Biosci. 86, 67-95 (1987)

12. Gyllenberg, M., Webb, G.F.: A nonlinear structured population model of tumor growth with quiescence. J. Math. Biol. 28, 671-694 (1990)

13. Heijmans, H.J.A.M.: On the stable size distribution of populations reproducing by fission into two unequal parts. Math. Biosci. 72, 19-50 (1984)

14. Inaba, H.: A semigroup approach to the strong ergodic theorem of the multistate stable population process. Math. Popul. Stud. 1, 49-77 (1988)

15. Kimmel, M., Darzynkiewicz, Z., Arino, O., Traganos, F.: Analysis of a cell cycle model based on unequal division of metabolic constituents to daughter cells during cytokinesis. J. Theor. Biol. 110, 637-664 (1984)

16. Koch, A.L., Holtje, J.V.: A physical basis for the precise location of the division site of rod-shaped bacteria: the central stress model. Microbiology 13, 3171-3180 (1995)

17. Mackey, M.C., Rudnicki, R.: Global stability in a delayed partial differential equation describing cellular replication. J. Math. Biol. 33, 89-109 (1994)

18. McKendrick, A.G.: Application of mathematics to medical problems. Proc. Edinb. Math. Soc. 14, 98130 (1926)

19. Lasota, A., Mackey, M.C.: Chaos, Fractals and Noise. Stochastic Aspects of Dynamics. Springer Applied Mathematical Sciences, vol. 97. Springer, New York (1994)

20. Metz, J.A.J., Diekmann, O. (eds.): The Dynamics of Physiologically Structured Populations. Springer Lecture Notes in Biomathematics, vol. 68. Springer, New York (1986)

21. Nooney, G.C.: Age distributions in dividing populations. Biophys. J. 7, 69-76 (1967)

22. Oldfield, D.G.: A continuity equation for cell populations. Bull. Math. Biophys. 28, 545-554 (1966)

23. Perthame, B.: Transport equations in biology. In: Frontiers in Mathematics. Birkhäuser, Basel (2007)

24. Laurençot, P., Perthame, B.: Exponential decay for the growth-fragmentation equation/cell-division equation. Commun. Math. Sci. 7(2), 503-510 (2009)

25. Pichór, K., Rudnicki, R.: Continuous Markov semigroups and stability of transport equations. J. Math. Anal. Appl. 249, 668-685 (2000)

26. Pichór, K.: Asymptotic stability of a partial differential equation with an integral perturbation. Ann. Pol. Math. 68, 83-96 (1998)

27. Rubinow, S.I.: A maturity time representation for cell populations. Biophys. J. 8, 1055-1073 (1968)

28. Rudnicki, R.: On asymptotic stability and sweeping for Markov operators. Bull. Pol. Acad. Sci., Math. 43, 245-262 (1995)

29. Rudnicki, R., Pichór, K.: Markov semigroups and stability of the cell maturity distribution. J. Biol. Syst. 8, 69-94 (2000)

30. Sharpe, F.R., Lotka, A.J.: A problem in age-distributions. Philos. Mag. 21, 435-438 (1911)

31. Trucco, E.: Mathematical models for cellular systems: The Von Foerster equation. Parts I and II. Bull. Math. Biophys. 27, 285-304 (1965) (See also pp. 449-470)

32. Von Foerster, H.: Some remarks on changing populations. In: Stohlman, F. (ed.) The Kinetics of Cellular Proliferation, pp. 382-407. Grune and Stratton, New York (1959) 